\title{
"Trabajo de paso" y condición juvenil en una cadena multinacional de supermercados en la ciudad de Rosario, Argentina
}

\author{
Jaime Guiamet
}

Este escrito forma parte de una investigación socioantropológica para mi tesis doctoral sobre los trabajadores jóvenes de una empresa multinacional de supermercados en la ciudad de Rosario, Argentina. En este artículo analizamos las nociones sobre el lugar que ocupa el trabajo en el supermercado entre estos jóvenes y las construcciones de sentido en torno a este empleo como un "trabajo de paso" o un "primer trabajo". En relación con esto, también nos aproximamos a los discursos y a las concepciones que circulan entre los trabajadores sobre su propia condición de jóvenes.

PALABRAS CLAVE: trabajo, juventud, supermercados, experiencias, antropología

\section{“Passing Job" and Juvenile Condition in a Multinational Chain of Supermarkets in Rosario, Argentina}

This paper is part of a socioanthropological research conducted for my Philosophiae Doctor on young workers of a multinational chain of supermarkets in the city of Rosario, Argentina. In this article we analyze the notions about the rol of the supermarket work among this young workers, and the constructions of sense they build about working at a supermarket as a "passing job" or a "first job". Related to this, we also make an approach to discourses and conceptions that circulate among workers about their young condition.

KEYWORDS: work, youth, supermarkets, experiences, anthropology

JAime Guiamet: Consejo Nacional de Investigaciones Científicas y Técnicas, Rosario, Argentina

jaimeguiamet@yahoo.com.ar

Desacatos, núm. 45, mayo-agosto 2014, pp. 71-84

Recepción: 14 de diciembre de 2012 / Aceptación: 4 de julio de 2013 


\section{INTRODUCCIÓN}

$\mathrm{E}$ ste trabajo forma parte de la investigación para mi tesis doctoral, que se centra en el problema de los procesos de trabajo, las relaciones laborales y la experiencia de trabajadores jóvenes en una cadena multinacional de supermercados. En este escrito trataremos un aspecto de la experiencia de los trabajadores relacionado con el lugar que le otorgan al trabajo en el supermercado entre sus trayectorias laborales, así como las concepciones que circulan sobre su "condición juvenil". La empresa en la que realizamos la investigación -a la que nombraremos $A$ para preservar el anonimato de los trabajadores- es de capital sudamericano y forma parte de un grupo empresarial mayor que opera en diversos países de Sudamérica, principalmente en el rubro minorista. La empresa inaugura el primer supermercado $A$ en Argentina en 1982 en la ciudad de Buenos Aires. En Rosario ${ }^{1}$ instala la primera sucursal en 2004 con la apertura de un sho-

pping en la zona norte de la ciudad, que lo constituye como el centro comercial más grande del interior del país en ese momento. La superficie del supermercado es de aproximadamente $8000 \mathrm{~m}^{2}$ y actualmente trabajan ahí casi 200 empleados.

El sector supermercadista ${ }^{2}$ tuvo una gran expansión en la década de 1990 en Argentina, en estrecha

\footnotetext{
${ }^{1}$ Rosario es uno de los principales nucleamientos urbanos de Argentina, con alrededor de un millón y medio de habitantes. A lo largo de la historia ha sido una ciudad con una importante dinámica comercial, industrial y estrechamente vinculada a la actividad agrícola pampeana a través del puerto. El proceso de desindustrialización iniciado en tiempos de la dictadura militar (1976) y su profundización en la década de 1990 produjo una fuerte crisis en la ciudad, que alcanzó una desocupación por encima de $20 \%$. En el periodo actual de "posconvertibilidad" ha recuperado su actividad económica fundamentalmente a partir del impulso de las exportaciones y el desarrollo de la agroindustria.

${ }^{2}$ Utilizaremos este término para referirnos al sector económico de las empresas de supermercados, no al sector mercantil en general. Según Abal (2005: 5), se considera "supermercado" a una superficie comercial - donde se comercializan alimentos pero también otros tipos de mercaderías-que excede los $350 \mathrm{~m}^{2}$ de superficie de ventas.
}

relación con las políticas neoliberales que se implementaron durante ese periodo. Con la administración de Menem (1989-1999) se produjo una transformación estructural a nivel social y económico en Argentina, cuyos principales rasgos fueron la desregulación laboral y comercial, la apertura financiera y la modificación del sector público mediante privatizaciones. Estos cambios fueron vehiculizados sobre todo a través de las leyes de Emergencia Económica (23696) y de Reforma del Estado (23697), así como mediante políticas económicas basadas en la paridad peso-dólar (Ley de Convertibilidad). En la rama supermercadista hubo concentración y extranjerización del capital favorecida fundamentalmente por dos factores vinculados entre sí: la apertura comercial y la ausencia de una política de defensa de la competencia (Rodríguez et al., 2010). Estos procesos generaron en el sector mercantil el quiebre masivo de pequeños y medianos comercios, con la consecuente pérdida de puestos de trabajo. Por otra parte, el ingreso de las grandes cadenas de supermercados implicó la imposición de condiciones de empleo precarias y nuevas formas de organizar el trabajo en el sector, lo que repercutió en particular en la ampliación de los horarios de atención — noche, fines de semana, etcétera-.

Por otro lado, en ese periodo tiene lugar una política de recambio de la mano de obra en el sector supermercadista, que continúa hasta la actualidad, en la que los trabajadores que tenían varios años de antigüedad en el sector son reemplazados por jóvenes que, por lo general, inician su vida laboral en ese trabajo. La contratación de jóvenes respondía a la intención de romper con ciertas tradiciones de lucha de la clase obrera, por lo que se buscó un trabajador que careciera de "prácticas de sociabilidad en tiempos de vigencia de una cultura del trabajo de resistencia, de oposición, de luchas colectivas y orgullo sindical" (Abal, 2004: 116). Además, debe tenerse en cuenta que la mayoría de los jóvenes que trabajan en supermercados no son jefes de familia y pueden tener una disponibilidad horaria más 
amplia que los adultos (Abal, 2004), acorde con la tendencia de estos grupos empresariales de imponer una flexibilización horaria cada vez mayor.

La presente investigación se enmarca en el área de la antropología del trabajo y en tanto tal parte de un enfoque metodológico que puede denominarse "enfoque antropológico", caracterizado por estudiar el trabajo en su cotidianeidad, en el lugar de producción, y que presta atención a las experiencias y los sentidos que le otorgan los sujetos sociales que lo desarrollan. Este enfoque pretende alejarse de perspectivas excesivamente centradas en los sentidos que construye el actor, que desatienden los procesos estructurales que atraviesan al mundo del trabajo. En lo que se refiere a las estrategias metodológicas aplicadas en el trabajo de campo con los trabajadores jóvenes de supermercados hemos recurrido de manera destacada a dos: entrevistas y observación con participación. Las entrevistas se asemejan a lo que Guber llama "entrevista antropológica o etnográfica, también conocida como entrevista informal o no directiva" (Guber, 1991: 205). Este tipo de entrevista se caracteriza por ser abierta, es decir, no se guía por un cuestionario cerrado y único para todos los sujetos, sino que se atiene al flujo de la conversación mediante la "no directividad", que se basa en técnicas como la atención flotante, la asociación libre y la categorización diferida. La observación con participación sería un punto intermedio entre la observación participante -modalidad clásica que traslada la cotidianeidad del investigador a la de los sujetos-y la observación no participante - que intenta minimizar la presencia del investigador en el campo-. El análisis de fuentes, como notas periodísticas sobre el tema, el Convenio Colectivo de Trabajo o el "Manual de inducción" de la empresa para los empleados, formó parte asimismo de nuestro marco metodológico.

Durante la investigación -que comienza en 2008 y se encuentra en curso en la actualidad - se han practicado 23 entrevistas a diferentes sujetos trabajadores del supermercado y diez observaciones orientadas fundamentalmente a conocer el proceso de trabajo. En este artículo retomaremos registros realizados con trabajadores que al momento de ser entrevistados se desempeñaban en el supermercado y con sujetos con los que hemos mantenido el vínculo una vez que han dejado ese empleo, ya que parte de nuestro interés es analizar cómo las concepciones se resignifican y se complejizan a lo largo del tiempo.

\section{ALGUNAS DISCUSIONES SOBRE LA JUVENTUD, SUS CATEGORIZACIONES Y SUS VÍNCULOS CON EL TRABAJO}

Como dijimos, el trabajo en el supermercado $A$ es principalmente "de jóvenes", característica que le imprime particularidades al trabajo en sí y a sus modos de desarrollarse. Debemos precisar la noción de "juventud", aunque sea de manera breve, para adquirir una comprensión más acabada del problema de estos trabajadores. La juventud en tanto concepto cobra fuerza a comienzos de la era industrial, en la cual se produce una ruptura entre la infancia y la adultez:

transformando en consecuencia un periodo de transición, que en la sociedad tradicional estaba claramente definido, en un tiempo de incertidumbre, objetivado en la sociedad moderna en procesos de búsqueda, diferenciación de preparación, principalmente a través de la escolarización secundaria, para llegar a ser adulto (Macri y Van Kemenade, 1993: 18).

Según Chaves (2009), será en las décadas de 1950 y 1960 en que se reconocerá al joven como actor social. La juventud se constituye como un grupo con demandas propias y una particular visión del mundo, la escuela y la familia ceden terreno ante los grupos de pares como agencia de socialización privilegiada. Dentro de las ciencias sociales existe cierto consenso en destacar que la juventud es una construcción sociohistórica, que se conforma de modos diferentes en el juego de relaciones sociales. 
Además, en la mayoría de las investigaciones se aclara "que la juventud no es una categoría definida exclusivamente por la edad y con límites fijos de carácter universal" (Chaves, 2009: 10). Sin embargo, no pueden pasarse por alto ciertos disensos. Para Bourdieu la juventud es "sólo una palabra", es decir, una construcción que cada sociedad impone arbitrariamente en el marco de una lucha en la que "las clasificaciones por edad vienen a ser una forma de imponer límites, de producir un orden en el cual cada quien debe mantenerse, donde cada quien debe ocupar su lugar" (Bourdieu, 2002: 164). En cambio, para otros autores "la juventud no es sólo un signo ni se reduce a los atributos 'juveniles' de una clase” (Margulis y Urresti, 2008: 28), y en esta línea proponen:

la idea de moratoria vital, que hace referencia a una característica cronológica de la juventud, un aspecto objetivante de su definición, que consiste en poseer mayor capital energético y sentirse (y estar) más alejado de la muerte que en otras generaciones (Chaves,

Otra noción desarrollada en los estudios sobre juventud es la idea de moratoria social. Este concepto se adhiere a la situación de ciertos jóvenes en la actualidad, en donde la juventud:

transcurriría entre el final de los cambios corporales que acaecen en la adolescencia y la plena integración a la vida social que ocurre cuando la persona forma un hogar, se casa, trabaja, tiene hijos. O sea, juventud sería el lapso que media entre la madurez física y la madurez social (Margulis y Urresti, citados en Chaves, 2009: 19).

Desde nuestra postura, la idea de moratoria social remite más a una construcción que intenta universalizar la situación de ciertos jóvenes que a una condición inherente a esa etapa de la vida. Pensamos que para comprender el concepto de juventud debe tenerse en cuenta la articulación con otras categorías de género, de clase social, étnicas, etc. También

destacamos el concepto de generación, que implica una variabilidad de acuerdo con la época y con la cultura en que se inscribe:

Cada generación puede ser considerada, hasta cierto punto, como parte de una cultura diferente, en la medida en que los más jóvenes incorporan en su socialización nuevos códigos y destrezas, lenguajes y formas de percibir, de apreciar, de clasificar y distinguir (Andreia, 2005: 5).

Hoy en día se dispone de una gran variedad de estudios que se preguntan por diversos problemas de los jóvenes en relación con el trabajo. Algunos se enfocan en las tendencias estructurales de la relación juventud-trabajo, como los que se ocupan de las trayectorias laborales de los jóvenes (Chitarroni y Jacinto, 2009; Jacinto et al., 2005). De acuerdo con Jacinto y colaboradores, quienes estudian las trayectorias educativo-laborales de jóvenes de sectores medios-bajos y bajos en Argentina:

la introducción de dimensiones subjetivas y miradas longitudinales sobre la relación con el trabajo se vinculan fuertemente a la comprensión de la "inserción" como un proceso. Antes, la inserción laboral de los jóvenes simplemente podía definirse como el momento en que un joven accedía a un empleo sobre la base de los saberes y/o credenciales adquiridas durante su escolaridad (Jacinto et al., 2005: 2).

Otras investigaciones, sobre todo desde una mirada cualitativa, se centran en fenómenos particulares de la juventud y su relación con el trabajo. Kornblit (2004) y Orellano y Rosendo (2004), por ejemplo, se interesan por los sentidos que tiene el trabajo para jóvenes próximos a egresar del nivel secundario, ya sea que trabajen o no. Ellos destacan una preocupación por la vinculación entre las transformaciones estructurales y la subjetividad de los jóvenes, y afirman que los ámbitos del trabajo y la educación atraviesan por una etapa de transición o de crisis. Por último, hemos rastreado otra tendencia de estudios que se preguntan por los sentidos sobre el trabajo o 
la construcción identitaria de los trabajadores jóvenes, en específico respecto de determinados sectores de trabajo, como el de Battistini y Wilkis (2005) en la industria automotriz, el de Svampa (2009) sobre las distintas generaciones de metalúrgicos y los de Abal (2004 y 2005) en torno a los jóvenes trabajadores de supermercados, entre los más importantes. ${ }^{3}$ En su mayoría, estos trabajos analizan la vinculación de los cambios ocurridos durante los últimos años a nivel social y en el plano de la producción con las transformaciones registradas en las "construcciones identitarias" de los sujetos, especialmente profundas en los jóvenes que ingresan al mercado de trabajo. Estos trabajos son útiles para nuestro enfoque ya que se aproximan a cómo se concretiza esta situación general de precariedad de la juventud en la práctica laboral cotidiana, que obviamente resulta particular de acuerdo con el sector y con el tipo de trabajo. En la investigación de Abal, quien trabaja el mismo sector que el nuestro, resalta el hecho de que las cadenas de supermercados se han volcado sistemáticamente a la contratación de jóvenes. Destaca una serie de características que promueven la contratación de estos sujetos:

en su mayoría los jóvenes no son jefes de hogar y por ello no poseen la responsabilidad primaria de sostener la familia. De esta forma sus ingresos laborales sólo complementan los gastos de hogar. En segundo lugar, son físicamente más sanos que los adultos por lo que pueden realizar un trabajo de fuerte intensidad librando "cheques de enfermedad" a futuro. Por último, tienden a tener una disponibilidad horaria mucho mayor que los adultos. Las empresas pueden flexibilizar sus jornadas y rotar permanentemente francos y turnos de trabajo (Abal, 2004: 117).

Según Palomino, la noción actual de "joven” en relación con el mundo laboral se sitúa en dos órdenes

\footnotetext{
${ }^{3}$ En este aspecto también podemos referir la ya mencionada investigación de Palomino (2002), que se centra en varios sectores de trabajo en donde se desempeñan los jóvenes y que abunda en los sentidos que tiene el trabajo para estos sujetos en la actualidad.
}

diferentes. Por un lado se piensa a la juventud como "problema", con base en concepciones como la de una "generación perdida", que alude a los fenómenos de pobreza y desempleo que afectan a una franja creciente de jóvenes. Por otro lado se los interpela como "solución", dentro de empresas que los solicitan para acelerar el cambio organizativo y el desplazamiento del personal de mayor edad (Palomino, 2002). Así, la relación entre juventud y trabajo es compleja y varía de acuerdo con el contexto sociohistórico en el que se la considere, pero también en función del sector social y los tipos de trabajo que desempeñen los jóvenes. En este caso analizaremos cómo jóvenes de un sector social en particular, ${ }^{4}$ en un trabajo en particular, construyen sus significaciones sobre las implicaciones de "ser joven" y trabajar en un supermercado.

\section{LAS EXPERIENCIAS DE LOS TRABAJADORES JÓVENES: ¿PASANDO POR EL TRABAJO?}

A continuación expondremos las experiencias de los trabajadores jóvenes en relación con el lugar que le otorgan a su empleo en el supermercado. Consideramos necesario describir las condiciones de trabajo más generales en el supermercado porque creemos que condicionan dichas experiencias. En lo

\footnotetext{
${ }^{4}$ Podemos afirmar que se trata de jóvenes provenientes de sectores medios. Por lo general residen en sus hogares de origen y sus padres se desempeñan en el sector formal de la economía como choferes de colectivos, obreros de fábricas, etc., y en algunos casos como trabajadores independientes que tenían su propio negocio - talleres mecánicos, pequeños locales de ropa, entre otros-. La mayoría de los jóvenes afirmaba que debían trabajar para costearse sus estudios - por lo general terciarios, y en algunos casos también universitarios- $-\mathrm{u}$ otras actividades que desarrollaban paralelamente al supermercado, pero no contribuían con una suma significativa a su hogar de origen - si bien no estudiaremos este aspecto, encontramos una diferencia de acuerdo con el género, ya que las mujeres decían aportar más a sus hogares-. Respecto del lugar de procedencia, la mayoría son rosarinos o de ciudades del Gran Rosario.
} 


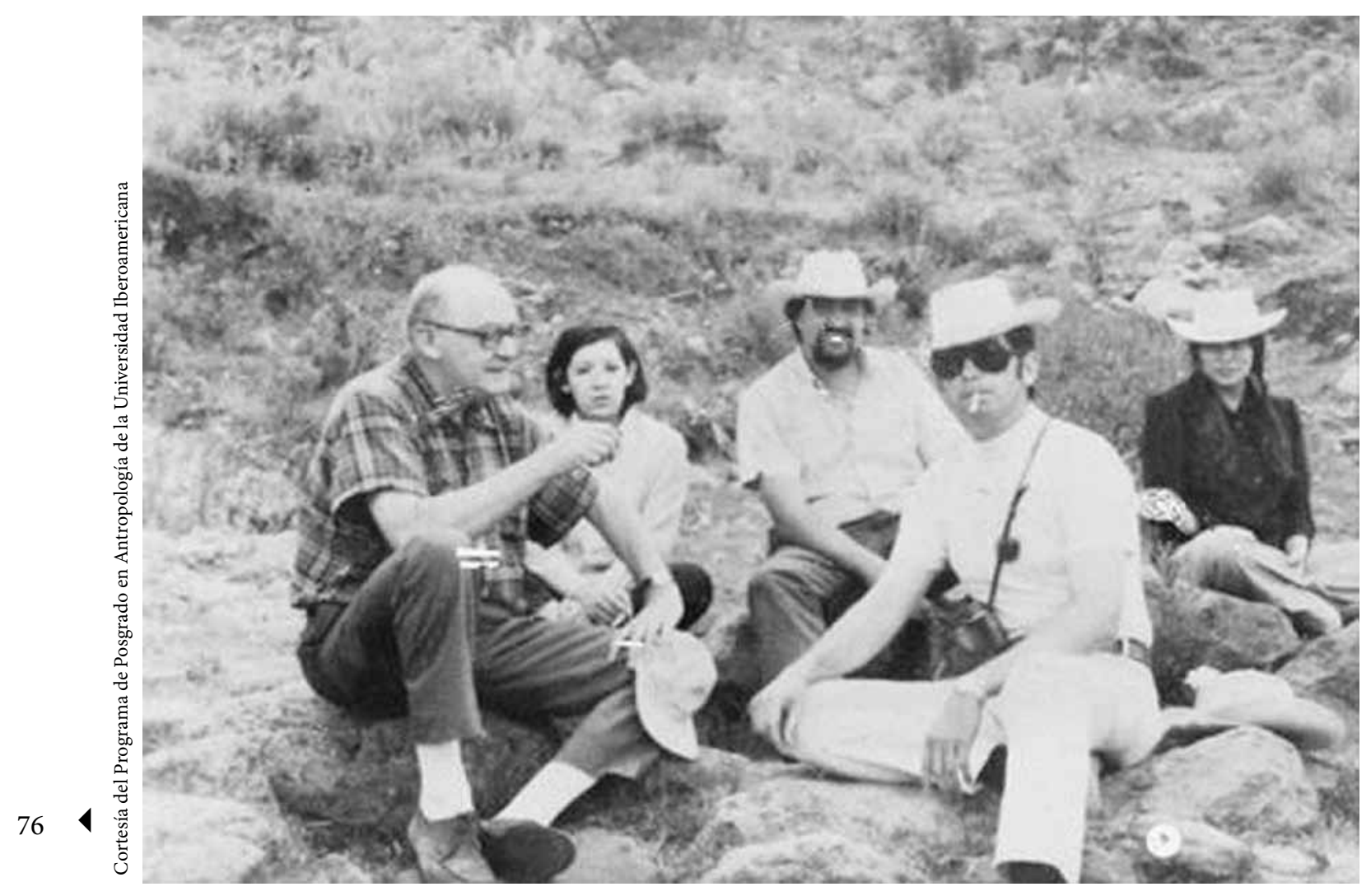

Ángel Palerm, Leticia Gándara, Andrés Fábregas, Pepe Lameiras y probablemente Luz María Mohar.

que corresponde a la situación contractual de los trabajadores, podemos diferenciar dos momentos. En una primera etapa, que va desde la apertura del supermercado, desde 2004 hasta 2008, éstos ingresaban contratados directamente por la empresa durante un periodo de prueba ${ }^{5}$ de tres meses, al cabo del cual se decidía si se los contrataba de manera definitiva. Después de 2009 se comenzó a subcontratarlos mediante las "agencias de empleo eventual", lo

\footnotetext{
${ }^{5}$ El periodo de prueba era utilizado por esta empresa para contratar un gran número de trabajadores en temporadas de mayor demanda, como las fiestas navideñas. Luego de que se terminaba este periodo, se prescindía de los trabajadores contratados sin pagar indemnización alguna por el despido. Consideramos que esta forma de contratación constituye un modo de flexibilizar la proporción de la fuerza de trabajo de acuerdo con las fluctuaciones de la demanda.
}

que le permitía a la empresa despedirlos luego de un periodo mayor, que en algunos casos se extendió hasta más de un año. También coexisten otras formas de subcontratación de trabajadores, como la externalización de áreas enteras - mantenimiento, seguridad, limpieza - y la subcontratación de empleados que reponen productos para determinadas empresas proveedoras del supermercado, siendo contratados por éstas.

Otra arista flexible del trabajo en el supermercado es el tiempo. Los contratos son de una variada carga horaria, la mayoría tienen jornada completa de 48 horas semanales, pero una proporción considerable de trabajadores cuenta con contratos de tiempo parcial, en particular de 20 o 30 horas semanales. Otro elemento es la extensión del horario de trabajo. Los jóvenes muchas veces laboran horas extra, que luego 
intercambian por jornadas de menos horas de trabajo otros días, lo que constituye una ilegalidad según la legislación laboral argentina. También ha existido un conflicto por la vigencia de la ley del descanso dominical, que la mayoría de las grandes empresas del sector comercial incumplen (Guiamet, 2012).

En lo que respecta al salario, éste se ajusta al monto básico estipulado en el convenio colectivo de los empleados de comercio. ${ }^{6}$ Vale aclarar que en Argentina el salario de los trabajadores del sector es uno de los más bajos. Sin embargo, la empresa $A$ considera algunos premios salariales vinculados principalmente al cumplimiento de los horarios, ya sea el "presentismo" - que constituye 12\% del básico y se otorga cuando el trabajador no llega tarde más de dos veces en el mes, siempre y cuando el retraso sea menor a 15 minutos - o el pago doble del trabajo los domingos y días feriados.

Las sanciones, el control y la vigilancia han sido estudiadas en otros trabajos (Guiamet, 2011). Aquí podemos afirmar que existe un fuerte control sobre las acciones de los trabajadores, que se expresa en una multiplicidad de sanciones disciplinarias y apercibimientos, en particular en relación con la atención al cliente, que se configura como una de las principales políticas de la empresa (Guiamet, 2011). Uno de los mecanismos por medio de los cuales se intenta controlar las actitudes de los trabajadores hacia los usuarios es una evaluación por parte de la empresa a cargo de un "cliente misterioso", que es una persona que se hace pasar por cliente. Así que cualquier cliente se convierte en un evaluador potencial, lo que genera una disposición constante de los trabajadores frente a éstos (Guiamet, 2011). En suma, como hemos afirmado, las condiciones de trabajo en la empresa pueden ser caracterizadas como "flexibles", tanto en relación con el tiempo de trabajo y con el salario, como con las situaciones contractuales.

\footnotetext{
${ }^{6}$ Convención Colectiva de Trabajo, Empleados de Comercio, 130/75.
}

Una de las políticas aplicadas por la empresa que constata esta "flexibilización laboral" es el recambio y la disminución de la mano de obra. Según Abal (2005), de 1998 a 2004 en el sector supermercadista en Argentina se advierte una disminución sostenida del personal ocupado por metro cuadrado de ventas que desciende $31.1 \%$. Por otra parte, la superficie total de ventas aumenta $46.8 \%$. En el periodo que va de abril de 2004 a abril de 2010 esta tendencia se mantiene, si bien la cantidad de personal ocupado por metro cuadrado de ventas desciende a menor ritmo, $10.5 \% .^{7}$ De igual modo, se observa un incremento sostenido en la intensificación del trabajo - ya que no se producen transformaciones tecnológicas importantes en el sector que justifiquen esta disminución de personal- que conlleva un aumento de la productividad por empleado. En la ciudad de Rosario, de acuerdo con Andreu y Rodríguez (2010: 22), la permanencia promedio de un empleado de supermercado es de tres años y la rotación anual es de $25 \%$.

Este problema se liga con los altos niveles de rotación laboral y de desocupación entre los jóvenes en comparación con otras franjas etarias. Palomino argumenta que el modelo de inserción laboral de los jóvenes no es independiente de los cambios registrados en el mercado de trabajo. En particular, la elevada tasa de desocupación abierta instalada a partir de mediados de la década pasada impacta sobre los jóvenes: los menores de 25 años representan entre 35 y $40 \%$ de los desocupados. En los últimos años, si bien la tasa de desocupación ha bajado, la tendencia persiste. Cifras proporcionadas por Chitarroni y Jacinto (2009) revelan que al primer trimestre de 2007 en el conjunto urbano documentado por la Encuesta Permanente de Hogares el desempleo de los jóvenes de 15 a 29 años duplicaba la tasa general y triplicaba la correspondiente a los adultos de 30 y más años.

\footnotetext{
${ }^{7}$ Estimación propia con base en datos del Instituto Nacional de Estadística y Censos (INDEC) para el periodo correspondiente.
} 
La empresa de hipermercados $A$ también presenta altos niveles de disminución y rotación de personal. En los ocho años de permanencia de esta cadena de tiendas en la ciudad, el número de trabajadores ha descendido aproximadamente a menos de 50\%. Así, la inestabilidad es una tendencia hegemónica provocada por la política empresarial en todo el sector supermercadista, lo que permite aprovechar una fuerte explotación del trabajo durante algunos años y renovar el personal constantemente. Sin embargo, esta concepción sobre la inestabilidad de ciertos trabajos no es privativa de la organización del trabajo en la empresa $A$, sino que a nivel social se ha construido cierto sentido común sobre la existencia de algunos trabajos que cumplirían la función de insertar al joven trabajador en el mercado laboral formal, donde se sobreentiende que la estadía será limitada. Incluso, estas primeras inserciones laborales son publicitadas por algunas empresas paradigmáticas en esta política como un aspecto positivo en las trayectorias de los trabajadores:

Siempre digo, es un trabajo de paso, un trabajo de paso. O el primer empleo. De hecho McDonald's hizo una propaganda con eso [...] aparecen tipos grandes, un ingeniero, otro empresario, retriunfadores en la vida, pero diciendo que "el primer trabajo fue McDonald's y ahí aprendí determinadas boludeces", muy terrible la propaganda (registro núm. 9, delegado sindical).

Sartelli anota respecto de los negocios de comida rápida que en éstos "se genera una dinámica en la que la relación con la empresa es establecida desde el comienzo como 'precaria', hecho facilitado por las normas de flexibilización laboral que se han desarrollado en la Argentina en los últimos años"(Sartelli,

\footnotetext{
8 "Boludo" o "boludeces" es típico del uso argentino del idioma español. En este contexto el término es utilizado para referirse a algo mínimo o pequeño. Más adelante, los trabajadores usarán el término "boludear" para referirse a prácticas de evasión del trabajo $o$ a acciones que denotan poca seriedad.
}

2001). Es sugestiva la lectura de la cartilla de información para los recién llegados, con frases que confirman esta inestabilidad, por ejemplo, "esperamos que tu estadía en la empresa sea agradable" (Sartelli, 2001: 36). Estas nociones contribuyen a la construcción de una idea sobre ciertos empleos como inestables, entre los que se incluyen los del sector supermercadista. Esto se articula con las políticas empresariales de rotación y disminución de personal, así como con políticas contractuales como la subcontratación de los trabajadores por agencias de empleo eventual. En este tenor, nos interesa destacar los sentidos que los propios trabajadores de la empresa $A$ construyen sobre la inestabilidad del trabajo en supermercados. Este empleo es conceptualizado como un "trabajo de paso", un "trabajo de jóvenes" y/o un "primer empleo":

Estos supermercados o hipermercados son trabajos de tiempo, tiempo corto, empezar, y después avanzar y seguir avanzando y no estancarte, si te estancás... Yo conozco locos, en Buenos Aires, que me contó, tienen 30, 35 años y siguen en lo mismo, feteando. Por el sueldo no se iban, no se iban qué sé yo... y tampoco te tenés que agarrar por eso (registro núm. 6, trabajador del sector fiambrería).

Ya cuando sos un poco viejo se te hace difícil conseguir un laburo de repositor (registro núm. 5, repositor de una empresa proveedora del supermercado).

Es un trabajo de paso, para agarrar un poco de experiencia y ya está, no para toda la vida (registro núm. 10 , trabajador del sector fiambrería).

En otros escritos (Guiamet, 2011) analizamos cómo estas significaciones implican una naturalización de la precariedad laboral y se entrelazan con la política empresarial de rotación laboral. De modo sintético, hemos caracterizado como inducidas ciertas renuncias - ya que no eran despidos ni renuncias voluntarias de los sujetos- que la empresa implementaba hacia trabajadores que tenían varios años de antigüedad. Básicamente, se les ofrecía una compensación económica mucho menor a la que 
les correspondería como indemnización en caso de despido a cambio de que presentaran la renuncia:

Eso es la otra cosa, arreglaron mucha gente, le dan plata para que renuncie, por el hecho de que ellos no se quieren ensuciar, mirá si no son vivos, no se quieren ensuciar, entonces arreglaron la mayoría $[. .$.$] vino$ un día mi jefe y dijo: "ustedes presten atención a la gente que echan". Porque es verdad, si vos después te ponías a ver, gente que ya estaba cansada del súper, gente que no quería trabajar más, gente que le fastidiaban muchas cosas, en teoría gente que directamente quería irse, porque no quería trabajar más y no veían la forma de cómo irse, ¿me entendés? Y ellos les daban un empujón, ellos vieron que no querían trabajar más y entonces les ofrecieron lo que les ofrecieron (registro núm. 20, trabajador del sector bazar).

Estas acciones caracterizadas como "arreglos" no sólo le reportaban un beneficio económico a la empresa, también pretendían disciplinar a los trabajadores que permanecían ahí. La noción de "trabajo de paso", por su parte, responde a los objetivos de la empresa de recambio de trabajadores, en específico los de mayor antigüedad, quienes, como veremos más adelante, se consideraban estancados en la empresa. A continuación examinaremos la relación entre las concepciones de los trabajadores sobre su propia juventud y esta forma de concebir el empleo en el supermercado. Algunos trabajadores hacían énfasis en el hecho de no necesitar el trabajo tanto como un adulto o la facilidad relativa de encontrar otro. En este caso destacaba que no tuvieran una familia a su cargo y que residieran en el hogar de origen, caso contrario de la situación de los trabajadores que tenían hijos, los cuales debían enfrentar de otro modo el trabajo e incluso comportarse de un modo diferente:

A veces tenés que bancarte ${ }^{9}$ a los encargados cuando vienen y te basurean y tenés que aguantártela. Más pasa con los que tienen hijos, ¿me entendés? Vos

\footnotetext{
${ }^{9}$ Este término refiere a la acción de "aguantar" o "soportar".
}

sabés que tenés que mantener un laburo porque tenés una boca que alimentar y no podés... Ahora, si sos solo, por ahí podés llegar a contestar todo (registro núm. 19, repositor de una empresa proveedora del supermercado).

Debemos aclarar que la categoría de juventud per se no nos permite atribuir estas características a los jóvenes en general, sino que debe pensarse cómo en estos casos particulares se articulan el origen social, el étnico, el género, etc., lo que puede contribuir a comprender la elaboración de estas nociones sobre la falta de necesidad del trabajo, sin dejar de tener en cuenta que se trata de trabajadores de tiempo completo o con jornadas de mínimo seis horas con turnos rotativos que dificultan el desarrollo de actividades extralaborales. Otro significado que se relaciona con estas nociones es pensar el trabajo como un medio que sirve para consumir ciertas mercancías, poder salir y realizar actividades con su propio ingreso, sin tener que pedir dinero a los padres:

La mayoría son jóvenes y muy pocos son los que tienen responsabilidades de familia como para seguir trabajando en un supermercado. O sea, lo hacen porque necesitan plata para salir, lo hacen porque necesitan plata para comprarse un auto, para mantenerse ellos, para no pedirle a los padres (registro núm. 12, delegado sindical).

El trabajo no lo hago por una necesidad, sino como ellos [sus padres] me dicen: "vos ahí tenés tu plata". Yo, fijate, del trabajo me pude comprar la moto ésa, me he comprado otras cosas, que en su momento no te digo que no me las han podido regalar pero qué mejor que darse un gusto uno mismo. Entonces yo digo: "hoy en día tengo algo mío" (registro núm. 20, trabajador del sector bazar).

Las ideas que hemos expuesto de los propios sujetos sobre su juventud se relacionan con una concepción que posee mucha fuerza a nivel social: la juventud como un periodo de moratoria social. La noción aparece asociada a aquella de que, a 
pesar de que se trata de jóvenes que trabajan no se dependería en una medida tan grande del trabajo como a edades mayores. Molina, en su investigación sobre los jóvenes trabajadores del delivery, afirma que también desde las concepciones de los trabajadores "la juventud es asociada a la ausencia de responsabilidades [...] un periodo variable signado por el abandono del colegio, los primeros trabajos y la ausencia de responsabilidad familiar" (Molina, 2005: 5-6). En el supermercado $A$, si bien se habla de jóvenes que tienen familia, la información disponible remite a un nivel que implica no sólo otra forma de desempeñarse en el trabajo, sino a otra dimensión de la necesidad de éste.

Por otra parte, nos preguntamos qué lugar ocupa el empleo en el supermercado en relación con estas concepciones sobre su condición juvenil. Este trabajo se piensa como un momento de transición entre el ingreso pleno al mundo laboral $-\mathrm{y}$ con éste a la adultez-y el egreso del nivel escolar medio. Emergen sentidos de los trabajadores que trazan analogías enmente en lo relativo a los comportamientos que los sujetos despliegan en ambos ámbitos. Las actitudes que principalmente aparecían relacionadas con dicha concepción de juventud eran prácticas de evasión del trabajo, como fumar en el baño y prolongar los tiempos de descanso, que los propios trabajadores criticaban y calificaban como "boludear":

\begin{abstract}
No, ahora están más estrictos, antes era la escuela secundaria, continuación [...] vos entrabas al baño y era un boliche, veías así nublado, todo cigarrillo, colillas de cigarrillo, es más, una vez tuve que ir al baño del vestuario porque no podía respirar, te ahogabas. Y lo cambiaron, ahora podés ir cinco minutos afuera para fumar pero vos ya no podés fumar adentro, te sancionan, o no sé qué harán. Bueno, pero eso es algo bueno. Ejemplo, para ir a merendar, antes se quedaban una hora, hora y media, boludeaban (registro núm. 26, trabajadora del sector ferretería).
\end{abstract}

Habrá que subrayar que las prácticas de evasión — "hacer cebo", "trabajo a reglamento", "trabajo a desgano", etc. - son comunes en todos los trabajos y han sido desplegadas de las más diversas maneras, muchas veces en el repertorio de acciones colectivas alternativas a las huelgas tradicionales. En este caso, dichas prácticas son interpretadas a la luz de la condición juvenil de los empleados y creemos que esto también tiene incidencia en que se considere como algo bueno el control sobre éstas. Hemos analizado situaciones en que prácticas similares se han articulado de un modo colectivo entre los trabajadores - sobre todo del sector de fiambrería- y que los propios sujetos han definido como huelgas en relación con un reclamo de aumento de horas de trabajo. Un repositor de mayor antigüedad, que trabaja para una empresa proveedora del supermercado, cercano a los 30 años, caracterizaba estas acciones como propias de "pendejos": 10

Trabajador (T): En $J$ por ahí es distinto. Los chicos son jóvenes en algunas cosas y por ahí no se dan cuenta.

Entrevistador (E): ¿En qué sentido?

T: Y por ahí ellos toman todo más a modo de joda [broma o divertimento]. Yo me acuerdo una vez que iban a hacer una movilización porque los sueldos eran bajos, porque querían la jornada, y me acuerdo que los chicos de fiambrería se reían y tomaban todo a modo de joda. Digo, si vas a luchar, luchá, porque de última es un bien para todos. Por ahí porque son pendejos como quien dice [...] están en la joda, por ahí les da todo lo mismo, no tenés la responsabilidad de decir: "tengo que mantener una casa". Yo cuando tenía la edad de ellos estaba más centrado en salir de trabajar, irme y salir de joda con mis amigos. Y me chupaba todo un huevo y lo tomaba a modo de risa. Pero si vos querés pelear por tener un mejor sueldo y eso, tenés que tener un pensamiento serio, ver de qué forma lo hacés y todo (registro núm. 5, repositor de empresa proveedora).

La condición juvenil de este trabajo y las analogías con la escuela secundaria también se veían reforzadas

\footnotetext{
${ }^{10}$ Se refiere a un modo denigrante de caracterizar a alguien joven o inmaduro.
} 
en los trabajadores que dejaban el supermercado y conseguían otro empleo. Uno de ellos compara su trabajo actual, en una fábrica de productos plásticos, con el del supermercado $A$, del que había sido despedido un año atrás. Aquí encontramos una dimensión del trabajo juvenil que va más allá del comportamiento de estos jóvenes y se vincula con concepciones más generales sobre el trabajo:

Trabajador (т): Era como una secundaria, porque era cumplir horarios e irte a tu casa. Pero yo no, no sentía responsabilidad, no lo tomaba como un trabajo, así como algo que estás haciendo fuerza o que no te gusta. Era más de cagarse de risa que otra cosa cuando empecé... Después, los últimos fue que no estaba el grupo, no encontraba la gente con qué llevarme, qué sé yo. Pero era como una secundaria, no lo tomaba como un laburo, así de: "Oh, me tengo que levantar, tengo que ir a trabajar", qué sé yo [...]. Y en la fábrica era diferente, había tipos grandes, gente que solamente iba a trabajar y te contaba la experiencia de que no tenían plata para darle de comer a los pibes, qué sé yo, y que se quedaban horas extras... Era más esclavizante, todo.

Entrevistador (E): ¿Y qué cosas te contaban?

T: No, que no llegaban a fin de mes, se hablaban otras cosas. Por ejemplo, yo con los compañeros del laburo del $A$ hablábamos de que: "Ay, me compré un jueguito de la Play". Y acá decían: "que tengo que comprarle los útiles a los pibes y no llego". Es difícil el trabajo, es diferente, es otra mentalidad (registro núm. 26, extrabajador de $A$ ).

Así, encontramos sentidos sobre lo que realmente constituye un trabajo como tal: hacer fuerza, ser esclavizante, etc., en oposición a la experiencia previa de la escuela secundaria o el trabajo en supermercados, más fáciles de sobrellevar a pesar de que impliquen

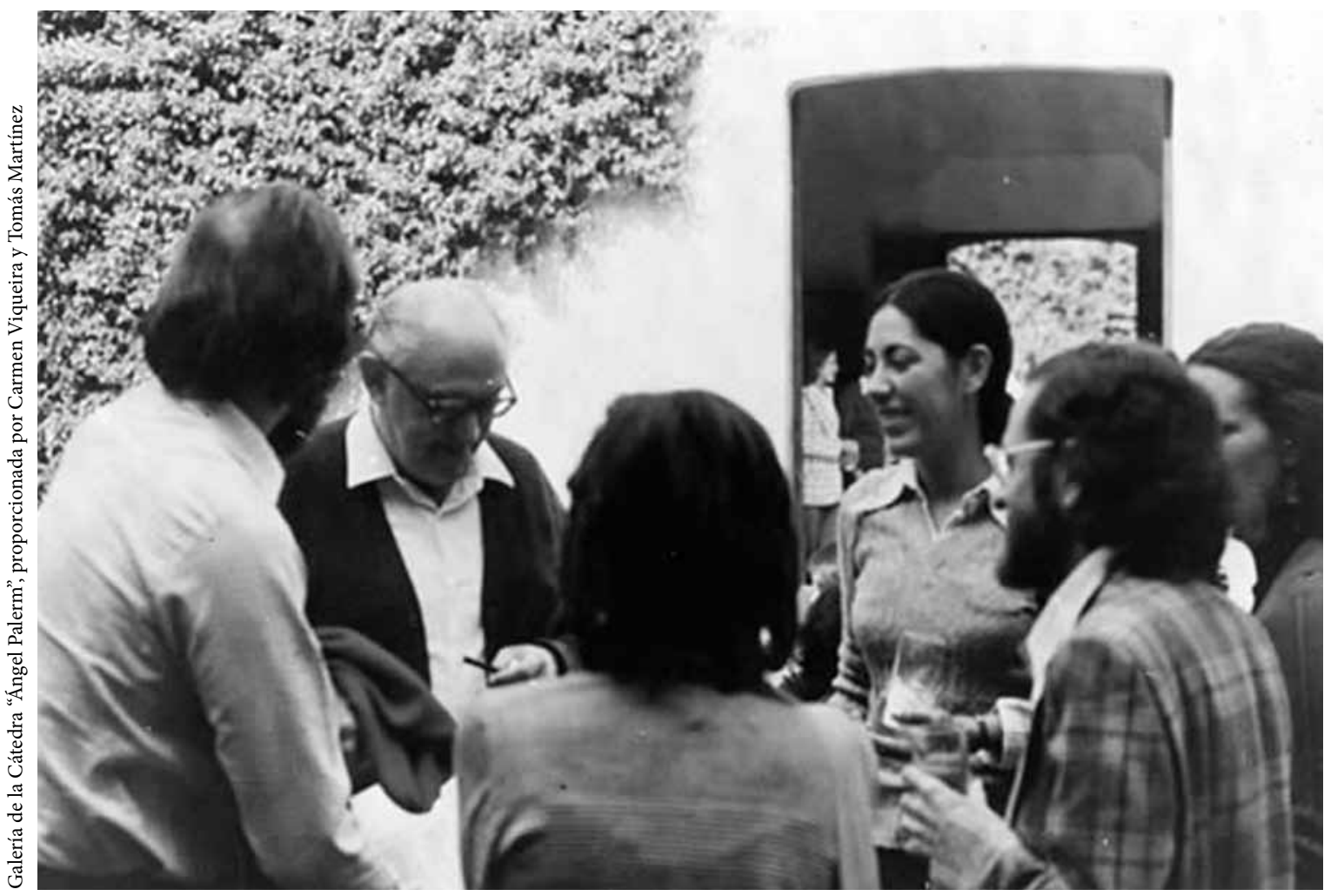

Casa Chata, 1973. 
cumplir horarios. Además aparece la vinculación con los compañeros y el hecho de compartir ciertos elementos como un condicionante de que un trabajo sea "más trabajo" que otro. Se hace referencia a las horas extra que se harían en la fábrica, aunque hemos observado que es muy frecuente que también se realicen en el supermercado, incluso sin paga o para reponer horas de otro día. La necesidad de hacer horas extra "para darle de comer a los hijos" es una diferencia sustantiva respecto de las realizadas en el supermercado, lo que posibilita que se visibilicen unas y no otras. Creemos necesario profundizar en estas experiencias posteriores al trabajo en el supermercado para comprender cómo se resignifica el primer trabajo a la luz de éstas.

En el caso anterior hemos retomado la experiencia de un trabajador luego de su paso por el supermercado, pero ¿qué sucede con quienes continúan laborando en la empresa $J$ más allá del tiempo estipulado? Preguntarnos por estos sujetos nos permite poner en cuestión la idea del trabajo de paso como de campo de modo casi ininterrumpido desde 2008 hasta la fecha encontramos sujetos que a lo largo del tiempo no han ascendido pero continúan en el mismo empleo, en especial porque han obtenido ciertos beneficios, como un margen mayor en la programación de sus bases horarias. Es interesante que la presencia duradera en el trabajo se manifieste por un lado como producto de dicha comodidad, pero a la vez como un estancamiento, idea que aparece ligada a la de adolescente, es decir, continuar trabajando en el supermercado sería un símbolo de inmadurez:

Estamos los que estamos estancados ahí, en una adolescencia eterna... [Hay veces] que vos tenés de largar a la mierda todo, pensás, ¿y después? O sí, salís a pelear de nuevo, pagar el derecho de piso en otra empresa [...]. Por lo menos acá ya estoy acomodado a la mañana, puedo flexibilizarme los horarios por si voy a tocar, laburo un franco y te lo devuelvo después. O sea, como que empiezo a pensar todas esas ventajas que ya logré con los años de estar ahí adentro y pagar de nuevo el derecho de piso entrando a otro lugar para

laburar no está bueno. Vos decís: "Me estoy cagando en todo lo que ya hice" (registro núm. 22, trabajador del sector de devoluciones).

Otro trabajador que percibe su permanencia en la empresa en términos de estancamiento habla de una necesidad en relación con el trabajo para poder llevar a cabo otros proyectos, el más importante de los cuales es el estudio. Esto nos permite retomar las ideas sobre la no necesidad del trabajo e intentar dinamizar estos sentidos que se complejizan entre los propios trabajadores. Podemos preguntarnos qué papel juega el hecho de que se trate de empleados con varios años de antigüedad, que están desde la apertura del supermercado hasta la actualidad, los que empiezan a mencionar la necesidad del trabajo:

\begin{abstract}
Sinceramente, en ciertos aspectos se puede decir que estamos estancados. Pero, cómo decir... uno tiene ciertos proyectos en su vida y si esto te ayuda a cumplir ese proyecto vos lo vas a seguir necesitando. Yo estoy tratando de terminar la carrera de enfermería y si este laburo me da la oportunidad de seguir estudiando, sinceramente, por el momento, me conviene estar ahí (registro núm. 22, trabajador del sector de recepción).
\end{abstract}

En este sentido queremos volver sobre lo que afirmábamos en relación con las renuncias inducidas, que operaban precisamente para desarticular esta comodidad, es decir, cambiando de horarios, de sector, de funciones a estos trabajadores de mayor antigüedad. En suma, nuestra aproximación al problema de las prácticas y significaciones sobre el trabajo en el supermercado nos da la pauta para complejizar la propia categoría social del trabajo de paso. En los casos en que se obtiene cierta estabilidad en el trabajo comienza a pensarse en una vinculación más duradera con la empresa. Sobre esta base es posible asimismo relativizar determinadas nociones sobre la vinculación actual de los jóvenes con el trabajo desde una perspectiva según la cual éstos no valorarían ni considerarían la posibilidad de una estabilidad en el trabajo. 


\section{CONCLUSIONES PARA SEGUIR REFLEXIONANDO}

En este texto hemos intentado visualizar cómo se categorizan estos trabajos desde varias dimensiones como empleos de jóvenes que están de paso. Desde la propia organización del trabajo, la patronal selecciona jóvenes en pos de facilitar la aplicación de distintas aristas de la flexibilización laboral, principalmente en lo que corresponde a la flexibilización horaria. También a nivel social se pretende naturalizar la idea de una trayectoria precaria e inestable en la que ciertos trabajos -en los que se sobreentiende que las condiciones serán peores que en los siguientes empleos- conforman un piso desde el cual iniciar una trayectoria ascendente. Así, la característica de pasaje que se les atribuye, o de iniciadores de jóvenes en el mundo laboral, encubre la sobreexplotación que dificulta que los trabajadores proyecten un futuro en la rama. En lo que respecta a las propias construcciones de sentido de los trabajadores sobre su condición juvenil, éstas se relacionan con ideas subyacentes sobre la caracterización de la juventud como un periodo de moratoria, en particular en torno a las ideas sobre la no necesidad del trabajo, al hecho de vivir en el hogar paterno, no tener una familia a cargo y trabajar para adquirir cierto nivel de consumo, entre otros. Los propios trabajadores asocian esta condición juvenil con diferentes aristas del trabajo: comportamiento, acciones colectivas, fin para el que se trabaja, etcétera.

Por último, tratamos de alejarnos de posiciones que muestran a los jóvenes como sujetos carentes de expectativas o de proyectos respecto del trabajo. Destacamos que emergían expectativas sobre la búsqueda y la valoración de estabilidad en el empleo en la empresa en algunos sujetos con mayor antigüedad, pero creemos que se debe fundamentalmente al logro de ciertas comodidades, entre las que sobresale tener un horario fijo. En principio, rescatamos la noción de "estancamiento" con la que los trabajadores caracterizan su situación cuando no coincide con las ideas sobre el carácter temporal o de paso del trabajo en supermercados. El problema de cómo estas concepciones buscan darle sentido a la relación entre los diversos proyectos de vida y las condiciones del mercado laboral actual queda como una línea para continuar la presente investigación.

\section{BIBLIOGRAFÍA}

Abal Medina, Paula, 2004, "Los dispositivos de control como mecanismos inhibidores de la identidad colectiva Un estudio de caso en grandes cadenas de supermercados", en Osvaldo Battistini (coord.), El trabajo frente al espejo. Continuidades y rupturas en los procesos de construcción identitaria de los trabajadores, Prometeo, Buenos Aires.

, 2005, Condiciones de trabajo y representación sindical. Un estudio de caso en una empresa supermercadista, Red Académica para el Diálogo Social, Buenos Aires.

Andreia de Souza, Diana, 2005, "El inmediatismo juvenil y su influencia en el mercado de trabajo. Realidad comparada entre Brasil y Argentina", en 7o. Congreso Nacional de Estudios del Trabajo, julio, Asociación Argentina de Especialistas de Estudios del Trabajo, Buenos Aires, en línea: <www.aset.org.ar>, consultado el 15 de julio de 2009.

Andreu, Patricia y Gloria Rodríguez, 2010, "Acerca de la relación entre la organización del trabajo y los procesos de salud-enfermedad en trabajadores de Rosario y su región. Estudio en los sectores supermercadista y de la salud estatal", en VI Jornadas de Investigación en Antropología Social, agosto, Buenos Aires.

Battistini, Osvaldo y Ariel Wilkis, 2005, "El valor del trabajo en las construcciones identitarias de un grupo de asalariados jóvenes", en Cuadernos de Antropología Social, núm. 22, Buenos Aires, pp. 55-75.

Bourdieu, Pierre, 2002, 'La 'juventud' no es más que una palabra”, en Pierre Bourdieu, Sociología y cultura, Grijalbo, México.

Chaves, Mariana, 2009, "Investigaciones sobre juventudes en la Argentina: estado del arte en ciencias sociales 1983-2006", en Papeles de Trabajo, Revista Electrónica del Instituto de Altos Estudios Sociales, Universidad 
Nacional de General San Martín, año 2, núm. 5, Buenos Aires.

Chitarroni, Horacio y Claudia Jacinto, 2009, “Precariedades, rotación y acumulación en las trayectorias laborales juveniles", en 9o. Congreso Nacional de Estudios del Trabajo, Asociación Argentina de Especialistas en Estudios del Trabajo, agosto, Buenos Aires, en línea: <www. aset.org.ar>, consultado el 23 de septiembre de 2012.

Convención Colectiva de Trabajo, Empleados de Comercio, 130/75, 1975, Buenos Aires.

Guber, Rosana, 1991, El salvaje metropolitano, Legasa, Buenos Aires.

Guiamet, Jaime, 2011, "En el comienzo del camino: relaciones laborales y construcción de subjetividad en trabajadores jóvenes de una cadena multinacional de supermercados", tesis de licenciatura en antropología, Universidad Nacional de Rosario, Rosario.

2012, "Acá no tenés vida: prácticas y significados vinculados con la flexibilización horaria de trabajadores jóvenes de una cadena multinacional de supermercados en la ciudad de Rosario", en Revista de la Escuela de Antropología, vol. XVIII, Rosario.

Instituto Nacional de Estadística y Censos (INDEC), "Encuesta a supermercados", en línea: <www.indec.mecon. ar/encuestaasupermercados $>$, consultado el 30 de mayo de 2010.

Jacinto, Claudia et al., 2005, “Jóvenes, precariedades y sentidos del trabajo", en 7o. Congreso Nacional de Estudios del Trabajo, julio, Asociación Argentina de Especialistas en Estudios del Trabajo, Buenos Aires, en línea: <www. aset.org.ar>, consultado el 23 de septiembre de 2012.

Kornblit, Analía, 2004, "Representaciones sociales y valores de los jóvenes argentinos en relación con el trabajo", en Monografías Virtuales, núm. 4, junio.

Macri, Mariela y Solange Van Kemenade, 1993, Estrategias laborales en jóvenes de barrios carenciados, Centro Editor de América Latina, Buenos Aires.
“Manual de inducción”, 2004, Gerencia de Recursos Humanos de la empresa $J$.

Margulis, Mario y Marcelo Urresti, 2008, "La juventud es más que una palabra”, en Mario Margulis (ed.), La juventud es más que una palabra, Biblos, Buenos Aires.

Molina Derteano, Pablo, 2005, "El futuro ya llegó. Jóvenes con trabajo de delivery en zonas populares", en 7o. Congreso Nacional de Estudios del Trabajo, julio, Asociación Argentina de Especialistas en Estudios del Trabajo, Buenos Aires, en línea: <www.aset.org.ar>, consultado el 15 de julio de 2009.

Orellano, Miguel y Ernestina Rosendo, 2004, Escuela, trabajo y transiciones juveniles: la década de los '90 como bisagra para pensar una relación conflictiva, $\mathrm{Fa}-$ cultad de Filosofía y Letras-Universidad de Buenos Aires (Cuadernos de Antropología Social, núm. 19), Buenos Aires.

Palomino, Héctor, 2002, “Los jóvenes y el trabajo: modelos de inserción y rupturas generacionales", en línea: $<$ www.crisolps.com $>$, consultado el 30 de octubre de 2011.

Rodríguez, Gloria et al., 2010, "Reestructuración productiva y procesos de salud-enfermedad en los trabajadores. Estudios de caso en Rosario y su región”, Informe Final, Programa de Promoción de la Investigación, Formación y Divulgación sobre Riesgos del Trabajo, Superintendencia de Riesgos del Trabajo, Rosario.

Sartelli, Eduardo, 2001, "Para comer una hamburguesa. El estudio de los procesos de trabajo, el debate Braverman y el fast food en Argentina”, en Revista Razón y Revolución, núm. 7.

Svampa, Maristella, 2009, "Identidades astilladas. De la patria metalúrgica al heavy metal", en Maristella Svampa (ed.), Desde abajo. La transformación de las identidades sociales, Biblos, Buenos Aires. 